Bull. Austral. Math. Soc.

VOL. 74 (2006) [385-392]

\title{
EXTENDED CESÀRO OPERATOR BETWEEN SOME HOLOMORPHIC FUNCTION SPACES
}

\author{
XIAOFEN LV
}

We characterize the boundedness and compactness of the extended Cesàro operator $T_{g}$ from $H^{\infty}$ to the mixed norm space and Bloch-type space (or little Bloch-type space), where $g$ is a given holomorphic function in the unit ball of $C^{n}$ and $T_{g}$ is defined by

$$
T_{g} f(z)=\int_{0}^{1} f(t z) \Re g(t z)(d t / t) .
$$

\section{INTRODUCTION}

Let $\mathbf{B}=\left\{z \in \mathbf{C}^{n} ;|z|<1\right\}$ be the unit ball of $\mathbf{C}^{n}$, and let $H(\mathbf{B})$ be the family of all holomorphic functions on $\mathrm{B}$. We denote by $H^{\infty}$ the space of all bounded functions in $H(\mathrm{~B}) . H^{\infty}$ is a Banach space under the norm

$$
\|f\|_{\infty}=\sup \{|f(z)| ; z \in \mathbf{B}\} .
$$

A positive continuous function $\varphi$ on $[0,1)$ is called normal if there are three constants $0 \leqslant \delta<1$ and $0<a<b$ such that

$$
\begin{aligned}
& \left(P_{1}\right) \quad \frac{\varphi(r)}{(1-r)^{a}} \text { is decreasing on }[\delta, 1) \text { and } \lim _{r \rightarrow 1^{-}} \frac{\varphi(r)}{(1-r)^{a}}=0 ; \\
& \left(P_{2}\right) \quad \frac{\varphi(r)}{(1-r)^{b}} \text { is increasing on }[\delta, 1) \text { and } \lim _{r \rightarrow 1^{-}} \frac{\varphi(r)}{(1-r)^{b}}=\infty .
\end{aligned}
$$

We extend it to $\mathrm{B}$ by $\varphi(z)=\varphi(|z|)$. For $f \in H(\mathbf{B})$ we set

$$
\|f\|_{p, q, \varphi}=\left\{\int_{0}^{1} M_{q}^{p}(f, r) \frac{\varphi^{p}(r)}{1-r} d r\right\}^{1 / p}, \quad 0<p<\infty,
$$

and

$$
\|f\|_{\infty, q, \varphi}=\sup _{0<r<1} M_{q}(\dot{f}, r) \varphi(r)
$$

Received 22nd May, 2006

Supported by the National Natural Science Foundation of China (No.10471039) and the Natural Science Foundation of Huzhou City (No.2005YZ02). The author would like to express her thanks to her supervisor, Prof. Zhangjian $\mathrm{Hu}$, for his encouragement.

Copyright Clearance Centre, Inc. Serial-fee code: 0004-9727/06 \$A2.00+0.00. 
Here

$$
\begin{aligned}
M_{q}(f, r) & =\left\{\int_{\partial \mathbf{B}}|f(r \zeta)|^{q} d \sigma(\zeta)\right\}^{1 / q}, \quad 0<q<\infty \\
M_{\infty}(f, r) & =\sup _{\zeta \in \partial B}|f(r \zeta)| .
\end{aligned}
$$

The mixed norm space $H_{p, q}(\varphi), 0<p, q \leqslant \infty$, is the space of all functions $f \in H(\mathrm{~B})$ for which $\|f\|_{p, q, \varphi}<\infty$. When $0<p=q<\infty, H_{p, q}(\varphi)$ is just the weighted Bergman space

$$
A_{a}^{p}(\varphi)=\left\{f \in H(\mathbf{B}):\|f\|_{A_{a}^{p}}=\left\{\int_{\mathbf{B}}|f(z)|^{p} \frac{\varphi^{p}(z)}{1-|z|} d v(z)\right\}^{1 / p}<\infty\right\} .
$$

A function $f \in H(\mathbf{B})$ is said to belong to the Bloch-type space $\mathcal{B}_{\varphi}$ if

$$
\|f\|_{\mathcal{B}_{\varphi}}=\sup _{z \in \mathbf{B}} \varphi(z)|\nabla f(z)|<\infty ;
$$

and it is said to belong to the little Bloch-type space $\mathcal{B}_{\varphi, 0}$ if

$$
\lim _{|z| \rightarrow 1} \varphi(z)|\nabla f(z)|=0 .
$$

Here

$$
\nabla f(z)=\left(\frac{\partial f}{\partial z_{1}}, \ldots, \frac{\partial f}{\partial z_{n}}\right)
$$

is the complex gradient of $f$. It is easy to check that both $\mathcal{B}_{\varphi}$ and $\mathcal{B}_{\varphi, 0}$ are Banach spaces under the norm $\|f\|_{\varphi}=|f(0)|+\|f\|_{\mathcal{B}_{\varphi}}$, and $\mathcal{B}_{\varphi, 0}$ is a closed subspace of $\mathcal{B}_{\varphi}$. When $\varphi(r)=1-r^{2}$ and $\varphi(r)=\left(1-r^{2}\right)^{1-\alpha}$ with $\alpha \in(0,1)$, two typical normal weights, the induced spaces $\mathcal{B}_{\varphi}$ are the Bloch space and Lipschitz type space, respectively.

Let $\mathbf{D}$ denote the open unit disc in the complex plane $\mathbf{C}$. For a holomorphic function $f(z)$ on $\mathbf{D}$ with Taylor expansion $f(z)=\sum_{j=0}^{\infty} a_{j} z^{j}$, the Cesàro operator acting on $f$ is

$$
C[f](z)=\sum_{j=0}^{\infty}\left(\frac{1}{j+1} \sum_{k=0}^{j} a_{k}\right) z^{j} .
$$

The behaviour of the operator $C[\cdot]$ have been studied extensively on various spaces of holomorphic functions (see $[5,6,7,8, \mathbf{1 1}, \mathbf{1 2}])$. A little calculation shows

$$
C[f](z)=\frac{1}{z} \int_{0}^{z} f(t)(\log (1 / 1-t))^{\prime} d t .
$$

Hence, on most holomorphic function spaces, $C[\cdot]$ is bounded if and only if the integral operator

$$
f \longmapsto \int_{0}^{z} f(t)(\log (1 / 1-t))^{\prime} d t
$$


is bounded. From this point of view it is natural to consider the extended Cesàro operator $T_{g}$ on $H(D)$ with holomorphic symbol $g$,

$$
T_{g} f(z)=\int_{0}^{z} f(t) g^{\prime}(t) d t
$$

The boundedness and compactness of this operator on Hardy spaces, Bergman spaces, Bloch-type spaces and Lipschitz spaces have been studied in $[\mathbf{1}, \mathbf{2}, \mathbf{1 0}]$.

For $f \in H(\mathrm{~B})$, the radial derivative of $f$ is

$$
\Re f(z)=\sum_{j=1}^{n} z_{j} \frac{\partial f(z)}{\partial z_{j}} .
$$

Given $g \in H(\mathbf{B})$, the operator $T_{g}$ on $H(\mathbf{B})$ is defined by

$$
T_{g} f(z)=\int_{0}^{1} f(t z) \Re g(t z) \frac{d t}{t}, \quad f \in H(\mathbf{B}), \quad z \in \mathbf{B} .
$$

It is trivial that (1.2) is just (1.1) when $n=1$. In the unit ball, $\mathrm{Hu}$ [3] got the characterisation on $g$ for which the induced extended Cesàro operator is bounded or compact on the Bergman space $L_{a, \omega}^{p}$, Zhang [13] studied the same problems between $\mathcal{B}_{\left(1-r^{2}\right)}$ and $\mathcal{B}_{\left(1-r^{2}\right) \text { q }}$ for $0<p, q<\infty$. And also, Hu discussed the boundedness and compactness of $T_{g}$ on the mixed norm space $H_{p, q}(\varphi)$, where $0<p, q \leqslant \infty$ (see [4]). The purpose of this work is to obtain the sufficient and necessary conditions on $g \in H(\mathbf{B})$, such that the operator $T_{g}: H^{\infty} \rightarrow H_{p, q}(\varphi)$ (respectively, $H^{\infty} \rightarrow \mathcal{B}_{\varphi}, H^{\infty} \rightarrow \mathcal{B}_{\varphi, 0}$ ) is bounded or compact.

In what follows, $C$ will stand for positive constants whose value may change from line to line but not depend on the functions in $H(\mathrm{~B})$. The expression $A \simeq B$ means $C^{-1} A \leqslant B \leqslant C A$.

\section{SOME PRELIMINARY RESULTS}

Lemma 2.1. ([4]) Let $0<p, q \leqslant \infty$ and $\varphi$ be normal. Then for any $f \in H(\mathrm{~B})$,

$$
\|f\|_{p, q, \varphi} \simeq|f(0)|+\left\{\int_{0}^{1} M_{q}^{p}(\Re f, r)\left(1-r^{2}\right)^{p} \frac{\varphi^{p}(r)}{1-r} d r\right\}^{1 / p} .
$$

LEMMA 2.2. ([9]) Let $\varphi$ be normal and $f \in H(\mathrm{~B})$. Then

(A) $f \in \mathcal{B}_{\varphi}$ if and only if $\sup _{z \in \mathbf{B}} \varphi(z)|\Re f(z)|<\infty$. Moreover,

$$
\|f\|_{\varphi} \simeq|f(0)|+\sup _{z \in \mathbf{B}} \varphi(z)|\Re f(z)| .
$$

(B) $f \in \mathcal{B}_{\varphi, 0}$ if and only if $\lim _{|z| \rightarrow 1} \varphi(z)|\Re f(z)|=0$. 
Lemma 2.3. Let $\varphi$ be normal, $0<p, q \leqslant \infty$ and $g \in H(\mathbf{B})$. Then $T_{g}: H^{\infty}$ $\rightarrow H_{p, q}(\varphi)$ (or $H^{\infty} \rightarrow \mathcal{B}_{\varphi}$ ) is compact if and only if for any bounded sequence $\left\{f_{j}\right\} \subseteq H^{\infty}$ which converges to 0 uniformly on any compact subset of $\mathbf{B}$, we have $\lim _{j \rightarrow \infty}\left\|T_{g} f_{j}\right\|_{p, q, \varphi}=0$ (or $\lim _{j \rightarrow \infty}\left\|T_{g} f_{j}\right\|_{\varphi}=0$ ).

Proof. It can be proved by Montel's Theorem and the definition of compact operator. The details are omitted here.

\section{MAIN ReSULTS}

THEOREM 3.1. Let $\varphi$ be normal, $0<p<\infty, 0<q \leqslant \infty$ and $g \in H(\mathrm{~B})$. Then the following statements are equivalent:
(A) $T_{g}: H^{\infty} \rightarrow H_{p, q}(\varphi)$ is bounded;
(B) $T_{g}: H^{\infty} \rightarrow H_{p, q}(\varphi)$ is compact;
(C) $g \in H_{p, q}(\varphi)$.

In this case, $\left\|T_{g}\right\| \simeq\|g-g(0)\|_{p, q, \varphi}$.

Proof: The implication $(B) \Rightarrow(A)$ is trivial.

(A) $\Rightarrow(\mathrm{C})$. Suppose $T_{g}: H^{\infty} \rightarrow H_{p, q}(\varphi)$ is bounded, by the fact that $g(z)=g(0)$ $+T_{g}(1)(z)$ we know $g \in H_{p, q}(\varphi)$. Moreover,

$$
\|g-g(0)\|_{p, q, \varphi}=\left\|T_{g}(1)\right\|_{p, q, \varphi} \leqslant C\left\|T_{g}\right\| .
$$

(C) $\Rightarrow(\mathrm{B})$. First, for $f, g \in H(\mathrm{~B})$, by direct calculation we see

$$
\Re\left(T_{g} f\right)(z)=f(z) \Re g(z) .
$$

Let $g \in H_{p, q}(\varphi),\left\{f_{j}\right\} \subseteq H^{\infty}$ satisfying $\left\|f_{j}\right\|_{\infty} \leqslant 1$. By Montel's Theorem, there exists some subsequence of $\left\{f_{j}\right\}$ converging to $f$ uniformly on any compact subset of $\mathbf{B}$. Without loss of generality, we suppose the subsequence is $\left\{f_{j}\right\}$ itself. Then $f \in H(\mathbf{B})$ and $\|f\|_{\infty}$ $\leqslant 1$. Hence

$$
M_{q}^{p}\left(\left(f_{j}-f\right) \Re g, r\right) \leqslant 2^{p} M_{q}^{p}(\Re g, r) .
$$

By $g \in H_{p, q}(\varphi)$, Lemma 2.1 and the dominated convergence theorem we obtain

$$
\int_{0}^{1} M_{q}^{p}\left(\left(f_{j}-f\right) \Re g, r\right)\left(1-r^{2}\right)^{p} \frac{\varphi^{p}(r)}{1-r} d r \rightarrow 0 \quad(j \rightarrow \infty) .
$$

Lemma 2.1 implies, as $j \rightarrow \infty$,

$$
\begin{aligned}
\left\|T_{g} f_{j}-T_{g} f\right\|_{p, q, \varphi}^{p} & \leqslant C \int_{0}^{1} M_{q}^{p}\left(\Re\left(T_{g} f_{j}-T_{g} f\right), r\right)\left(1-r^{2}\right)^{p} \frac{\varphi^{p}(r)}{1-r} d r \\
& =C \int_{0}^{1} M_{q}^{p}\left(\left(f_{j}-f\right) \Re g, r\right)\left(1-r^{2}\right)^{p} \frac{\varphi^{p}(r)}{1-r} d r \\
& \rightarrow 0 .
\end{aligned}
$$


Therefore, $T_{g}: H^{\infty} \rightarrow H_{p, q}(\varphi)$ is compact.

Furthermore, for any $f \in H^{\infty}$, Lemma 2.1 yields

$$
\begin{aligned}
\left\|T_{g} f\right\|_{p, q, \varphi}^{p} & \leqslant C \int_{0}^{1} M_{q}^{p}(\Re g, r) \sup \left\{|f(z)|^{p} ;|z|=r\right\}\left(1-r^{2}\right)^{p} \frac{\varphi^{p}(r)}{1-r} d r \\
& \leqslant C\|g-g(0)\|_{p, q, \varphi}^{p}\|f\|_{\infty}^{p} .
\end{aligned}
$$

This, together with (3.1), means $\left\|T_{g}\right\| \simeq\|g-g(0)\|_{p, q, \varphi}$. The proof is completed.

REMARK. When $p=\infty$, the implication $(\mathrm{C}) \Rightarrow(\mathrm{B})$ does not hold for any $0<q \leqslant \infty$ in general. For example, we let $n=1$, and choose some $g$ and $\varphi$ satisfying (C) but $T_{g}: H^{\infty} \rightarrow H_{p, q}(\varphi)$ is not compact. In fact, set $g(z)=\left(z /(1-z)^{1+(1 / q)}\right)$, where $z \in \mathbf{D}$ and $0<q \leqslant \infty, \varphi(r)=1-r$. Then for $0<q<\infty$,

$$
M_{q}(g, r)=\left\{\frac{1}{2 \pi} \int_{0}^{2 \pi} \frac{r^{q} d \theta}{\left|1-r e^{i \theta}\right|^{q+1}}\right\}^{1 / q} \simeq \frac{1}{1-r}
$$

as $r \rightarrow 1^{-}$. For $q=\infty$,

$$
\sup _{0 \leqslant \theta<2 \pi} \frac{r}{\left|1-r e^{i \theta}\right|} \simeq \frac{1}{1-r} \text { as } r \rightarrow 1^{-} .
$$

Hence, for $0<q \leqslant \infty$ and $1 / 2 \leqslant r<1$,

$$
M_{q}(g, r) \varphi(r) \simeq \frac{1}{1-r} \varphi(r)=1 .
$$

Write $f_{j}(z)=z^{j}, z \in \mathbf{D}$. Then $\left\|f_{j}\right\|_{\infty} \leqslant 1$ and $\left\{f_{j}\right\}$ converges to 0 uniformly on any compact subset of D. However, for each $j$, Lemma 2.1 and $g(0)=0$ yield

$$
\begin{aligned}
\left\|T_{g} f_{j}\right\|_{\infty, q, \varphi} & \simeq \sup _{0<r<1} M_{q}\left(\Re T_{g}\left(f_{j}\right), r\right)\left(1-r^{2}\right) \varphi(r) \\
& =\sup _{0<r<1} r^{j} M_{q}(\Re g, r)\left(1-r^{2}\right) \varphi(r) \\
& \geqslant C \sup _{(1 / 2) \leqslant r<1} r^{j} M_{q}(g, r) \varphi(r) \\
& \geqslant C \lim _{r \rightarrow 1^{-}} r^{j}=C,
\end{aligned}
$$

where the constant $C$ is independent of $j$.

THEOREM 3.2. Let $\varphi$ be normal and $g \in H(\mathrm{~B})$. Then the following statements are equivalent:
(A) $T_{g}\left(H^{\infty}\right) \subseteq \mathcal{B}_{\varphi, 0}$;
(B) $T_{g}: H^{\infty} \rightarrow \mathcal{B}_{\varphi, 0}$ is bounded;
(C) $T_{g}: H^{\infty} \rightarrow \mathcal{B}_{\varphi}$ is compact;
(D) $T_{g}: H^{\infty} \rightarrow \mathcal{B}_{\varphi, 0}$ is compact; 
(E) $g \in \mathcal{B}_{\varphi, 0}$

In this case, $\left\|T_{g}\right\| \simeq \sup _{z \in \mathbf{B}} \varphi(z)|\Re g(z)|$.

Proof: The implications $(B) \Rightarrow(A)$ and $(D) \Rightarrow(C)$ are obvious.

(B) $\Rightarrow(\mathrm{E})$. Suppose $T_{g}: H^{\infty} \rightarrow \mathcal{B}_{\varphi, 0}$ is bounded, then $g=g(0)+T_{g}(1) \in \mathcal{B}_{\varphi, 0}$. Furthermore,

$$
\sup _{z \in \mathbf{B}} \varphi(z)|\Re g(z)| \simeq\|g-g(0)\|_{\varphi}=\left\|T_{g}(1)\right\|_{\varphi} \leqslant C\left\|T_{g}\right\| .
$$

$(\mathrm{E}) \Rightarrow(\mathrm{B})$. Let $g \in \mathcal{B}_{\varphi, 0}$, then for any $f \in H^{\infty}, T_{g} f \in \mathcal{B}_{\varphi, 0}$. Moreover,

$$
\left\|T_{g} f\right\|_{\varphi} \simeq \sup _{z \in \mathbf{B}} \varphi(z)|\Re g(z)||f(z)| \leqslant\|f\|_{\infty} \sup _{z \in \mathbf{B}} \varphi(z)|\Re g(z)| \leqslant C\|f\|_{\infty} .
$$

This, together with (3.2), shows $\left\|T_{g}\right\| \simeq \sup _{z \in \mathbf{B}} \varphi(z)|\Re g(z)|$.

(A) $\Rightarrow$ (B). Suppose $\left\{f_{j}\right\} \subseteq H^{\infty}, f \in H^{\infty}$ and $h \in \mathcal{B}_{\varphi, 0}$ satisfying $\lim _{j \rightarrow \infty}\left\|f_{j}-f\right\|_{\infty}=0$ and $\lim _{j \rightarrow \infty}\left\|T_{g} f_{j}-h\right\|_{\varphi}=0$. Then

$$
f_{j}(z) \rightarrow f(z) \quad(j \rightarrow \infty), \quad z \in \mathbf{B}
$$

And

$$
\left|T_{g} f_{j}(0)-h(0)\right|+\sup _{z \in \mathbf{B}} \varphi(z)\left|\Re g(z) f_{j}(z)-\Re h(z)\right| \rightarrow 0 \text { as } j \rightarrow \infty .
$$

So $h(0)=0$ and for every $z \in \mathbf{B}$

$$
f_{j}(z) \Re g(z) \rightarrow \Re h(z) \quad(j \rightarrow \infty)
$$

By (3.3), we have

$$
\lim _{j \rightarrow \infty} f_{j}(z) \Re g(z)=f(z) \Re g(z), \quad z \in \mathbf{B} .
$$

Thus, (3.4) and (3.5) imply $f(z) \Re g(z)=\Re h(z)$. Therefore,

$$
h(z)=\int_{0}^{1} \Re h(t z) \frac{d t}{t}=\int_{0}^{1} f(t z) \Re g(t z) \frac{d t}{t}=\left(T_{g} f\right)(z) .
$$

Consequently, $T_{g}: H^{\infty} \rightarrow \mathcal{B}_{\varphi, 0}$ is a closed operator. By the closed graph theorem, $T_{g}: H^{\infty} \rightarrow \mathcal{B}_{\varphi, 0}$ is bounded.

$(\mathrm{C}) \Rightarrow(\mathrm{E})$. Suppose $g \notin \mathcal{B}_{\varphi, 0}$. Then there would be some $\varepsilon_{0}>0$ and some sequence $\left\{z^{j}\right\} \subseteq \mathbf{B}$ satisfying $\lim _{j \rightarrow \infty}\left|z^{j}\right|=1$, but for each $j, \varphi\left(z^{j}\right)\left|\Re g\left(z^{j}\right)\right|>\varepsilon_{0}$. Set

$$
f_{j}(z)=\frac{1-\left|z^{j}\right|^{2}}{1-\left\langle z, z^{j}\right\rangle}, \quad z \in \mathbf{B}
$$


It is easy to check that $\left\{f_{j}\right\}$ is a bounded sequence in $H^{\infty}$ and $f_{j} \rightarrow 0$ uniformly on any compact subset of $\mathrm{B}$ as $j \rightarrow \infty$. Since $T_{g}: H^{\infty} \rightarrow \mathcal{B}_{\varphi}$ is compact, by Lemma 2.3,

$$
\left\|T_{g} f_{j}\right\|_{\varphi} \rightarrow 0 \quad(j \rightarrow \infty)
$$

On the other hand,

$$
\begin{aligned}
\left\|T_{g} f_{j}\right\|_{\varphi} & \simeq\left|T_{g} f_{j}(0)\right|+\sup _{z \in \mathbf{B}} \varphi(z)|\Re g(z)|\left|f_{j}(z)\right| \\
& \geqslant \varphi\left(z^{j}\right)\left|\Re g\left(z^{j}\right)\right|\left|f_{j}\left(z^{j}\right)\right| \\
& \geqslant \varphi\left(z^{j}\right)\left|\Re g\left(z^{j}\right)\right| \\
& \geqslant \varepsilon_{0} .
\end{aligned}
$$

This is a contradiction to (3.6).

(E) $\Rightarrow$ (D). Suppose $g \in \mathcal{B}_{\varphi, 0}$, then for any $f \in H^{\infty}, T_{g} f \in \mathcal{B}_{\varphi, 0}$. And also, for every $\varepsilon>0$, there exists some $r>0$ such that

$$
\varphi(z)|\Re g(z)|<\varepsilon \quad \text { whenever }|z|>r .
$$

Let $\left\{f_{j}\right\}$ be any bounded sequence in $H^{\infty}$, say $\left\|f_{j}\right\|_{\infty} \leqslant 1$ and $f_{j} \rightarrow 0$ uniformly on any compact subset of $\mathbf{B}$ as $j \rightarrow \infty$. Then for the above $\varepsilon$, there is a positive integer $J$ such that for $|z| \leqslant r$ and $j>J$,

$$
\left|f_{j}(z)\right|<\frac{\varepsilon}{\|g\|_{\varphi}+1}
$$

Thus, combining (3.7) and (3.8), we have

$$
\left\|T_{g} f_{j}\right\|_{\varphi} \simeq \sup _{z \in \mathbf{B}} \varphi(z)\left|\Re g(z) \| f_{j}(z)\right|<\varepsilon \quad \text { if } j>J .
$$

By Lemma 2.3, $T_{g}: H^{\infty} \rightarrow \mathcal{B}_{\varphi, 0}$ is compact. The proof is completed.

\section{REFERENCES}

[1] A. Aleman and J. Cima, 'An integral operator on $H^{p}$ and Hardy's inequality', J. Anal. Math. 85 (2001), 157-176.

[2] A. Aleman and A.G. Siskakis, 'Integration operators on Bergman spaces', Indiana Univ. Math. J. 46 (1997), 337-356.

[3] Z.J. Hu, 'Extended Cesàro operators on Bergman spaces', J. Math. Anal. Appl. 296 (2004), 435-454.

[4] Z.J. Hu, 'Extended Cesàro operators on mixed norm spaces', Proc. Amer. Math. Soc. 131 (2003), 2171-2179.

[5] J. Miao, 'The Cesàro operator is bounded on $H^{p}$ for $0<p<1$ ', Proc. Amer. Math. Soc. 116 (1992), 1077-1079. 
[6] Ch. Pommerenke, 'Schlichte funktionen und analytische funktionen von beschrankter mittlerer oszilation', Comment Math. Helv. 52 (1977), 591-602.

[7] A.G. Siskakis, 'Composition semigroups and the Cesàro operator on $H^{p}, J$. London Math. Soc. (2) 36 (1987), 153-164.

[8] A.G. Siskakis, 'On the Bergman space norm of the Cesàro operator', Arch. Math. (Basel) 67 (1996), 312-318.

[9] X.M. Tang, 'Extended Cesàro operators between Bloch-type spaces in the unit ball of $\mathrm{C}^{n}$ ', J. Math. Anal. Appl. (2006) (to appear).

[10] S.S. Wang and Z.J. Hu, 'Extended Cesàro operators on Bloch-type spaces', (Chinese), Chinese Ann. Math. Ser. A 26 (2005), 613-624.

[11] J. Xiao, 'Cesàro operators on Hardy, BMOA and Bloch spaces', Arch. Math. (Basel) 68 (1997), 398-406.

[12] J. Xiao and H. Tan, ' $p$-Bergman spaces $\alpha$-Bloch spaces little $\alpha$-Bloch spaces and Cesàro means', (Chinese), Chinese Ann. Math. Ser. A 19 (1998), 187-196.

[13] X.J. Zhang, 'Weigted Cesàro operators on Dirichlet type spaces and Bloch type spaces of $\mathrm{C}^{n}$ ', (Chinese), Chinese. Ann. Math. Ser. A 26 (2005), 139-150.

Department of Mathematics

Huzhou Teachers College

Huzhou

Zhejiang, 313000

Peoples Republic of China

e-mail: Ixf00411@126.com 